\title{
A Commentary on the Significance of Stresscoat and Split-line Patterns on Bone
}

\author{
F. GAYNOR EVANS \\ Department of Anatomy, The University of Michigan, Ann Arbor, Michigan
}

\begin{abstract}
Stresscoat," an industrial technique for showing sites of highest tensile strain and failure in machine parts, has been used for similar purposes in intact living and cadaver bones. Stresscoat cracks only arise from tensile strain in the underlying material and always lie transverse to the direction of the strain. Stresscoat and split-line patterns do not represent stress trajectories. A trajectorial diagram can not be drawn for a bone because a bone is a three dimensional porous body composed of heterogeneous anisotropic material. Split-lines show the "grain" of bone and may be related to its vascularity or growth. The mechanical significance of split-lines is not clear.
\end{abstract}

Although bone has been studied by means of the stresscoat and the split-line techniques, only Evans and Goff ('57) used both techniques and illustrated both types of pattern on the same bone. The stresscoat patterns we obtained have been criticized by Tappen (Am. J. Phys. Anthrop., 22, '64) because they do not aid in explaining the orientation of split-line patterns in compact bone! The editor has kindly allowed me this opportunity to discuss Tappen's criticism.

"Stresscoat" is the trade name for a brittle resinous lacquer, developed by deForest and Ellis ('40), that, in spite of its name, cracks in response to tensile strain in the material upon which the lacquer has been sprayed. The sensitivity of stresscoat lacquer is calibrated in inches per inch. For example, the sensitivity of the stresscoat lacquer used in our experiments was $0.0007 \mathrm{in} / \mathrm{in}$. This means that when the material upon which the lacquer is sprayed elongates 0.0007 of an inch the overlying lacquer will crack. The elongation (tensile strain) of the material which is coated with stresscoat lacquer can occur from bending or from pulling. The cracks forming a stresscoat pattern lie transverse to the direction of the tensile strain in the underlying material and first appear in the area of highest tensile strain where failure will occur with sufficient load. The stresscoat pattern thus shows the site of highest tensile strain, the general distribution of tensile strain, and the direction of tensile strain in the body being tested. The stresscoat technique is used in industry to determine sites of highest tensile strain where failure can be expected in airplane, automobile, and machine parts. The technique of using stresscoat, the principles upon which it is based, and the significance of stresscoat patterns on the bones of living animals as well as those from cadavers have been thoroughly described, discussed, and illustrated in my monograph STRESS AND STRAIN IN Bones ('57), a reference cited by Tappen.

The stresscoat technique should not be expected to aid in explaining the orientation of split-lines in bone. As we (Evans and Goff, loc. cit.) clearly stated, "The stresscoat method is excellent for studying the mechanical behavior of a body under various types of loading but is of relatively little direct use in analysing the composition of the material composing the body. Thus, a piece of wood, bone or metal of similar shape and dimensions would, if similarly loaded, have the same type of stresscoat pattern." The only difference would be the magnitude of the load, which would vary with the physical properties of the material composing the body, required to produce the stresscoat pattern.

We also pointed out that "The patterns obtained by use of stresscoat are strain, not stress, patterns and only arise from tensile strain in the material upon which the lacquer has been sprayed." The tensile strain causing the cracks in the stress- 
coat lacquer is produced by bending of the material in the region where the pattern is found.

A bone like the femur may be subjected to a variety of force systems during lifebending, twisting, impacts and so on. It should be understood that our stresscoat experiments represented a vertical columntype loading roughly like that occurring when a person is standing erect. If the load is large enough the femur bends like a slender column or bowshaft. Bending of the femur under static vertical loading (fig. 1) has been clearly demonstrated by Evans and Lissner ('48). During bending
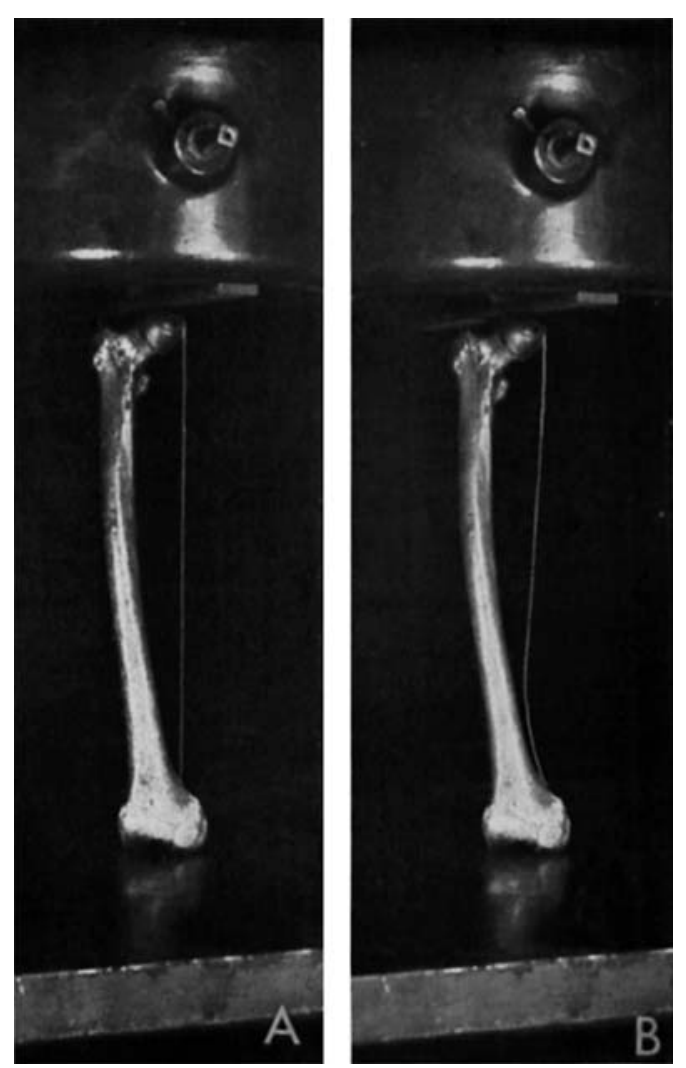

Fig. 1 Bending of a human femur under load (from Evans and Lissner, Anat. Rec., 100 , '48).

A. Unloaded femur in a materials-testing machine. Note taut white thread.

B. Same bone under a load of $650 \mathrm{lbs}$. Note slackness of white thread as a result of bending of the bone. When the load was removed the bone returned to its pretest dimensions. The thread became taut again. the convex side of the femur (superior aspect of the neck and latero-anterior aspect of the shaft) is elongated through tension while the concave side of the bone (inferior aspect of the neck and medioposterior aspect of the shaft) is shortened through compression. The elongation (tensile strain) in the bone causes the overlying stresscoat lacquer to crack thus giving rise to stresscoat patterns showing the distribution of tensile strain produced by bending of the femur.

Our experiments consisted of placing a stresscoated femur, oriented similar to the position of the bone when one stands erect with the heels together, in a Riehle 5,000 lb. capacity materials testing machine calibrated to an accuracy of $\pm 1 \%$ and slowly applying a load to the head of the bone until a stresscoat pattern was obtained. "Leaving the femur under load for several minutes gave the lacquer an opportunity to flow so that when the load was gradually removed and the bone slowly returned to its original dimensions cracks appeared on the inferior aspect of the neck and the medial aspect of the shaft. These cracks, constituting the second stresscoat pattern, actually arose from tensile strain produced by stretching of the bone as it returned to its pretest dimensions. However, this second pattern lies in the regions of the bone subjected to compressive strain during loading and thus indicates the location and the extent of the compressive strain in the bone when loaded" (fig. 2).

In comparing the orientation of cracks forming a stresscoat pattern on the shaft of the femur with that of split-lines in the same region (fig. 3 ) Tappen notes that we did not discuss why the stresscoat cracks, which are transverse to the long axis of the bone, "are at virtual right angles to the major orientation of split-lines throughout the shaft." The stresscoat cracks must be transverse to the long axis of the bone because they only arise from tensile strain in the underlying material and always lie transverse to the direction of the strain. Under the conditions of our tests it is obvious that tensile strain, in the direction of the long axis of the femoral neck and shaft, was created by bending of the femur under the superimposed load. The magni- 

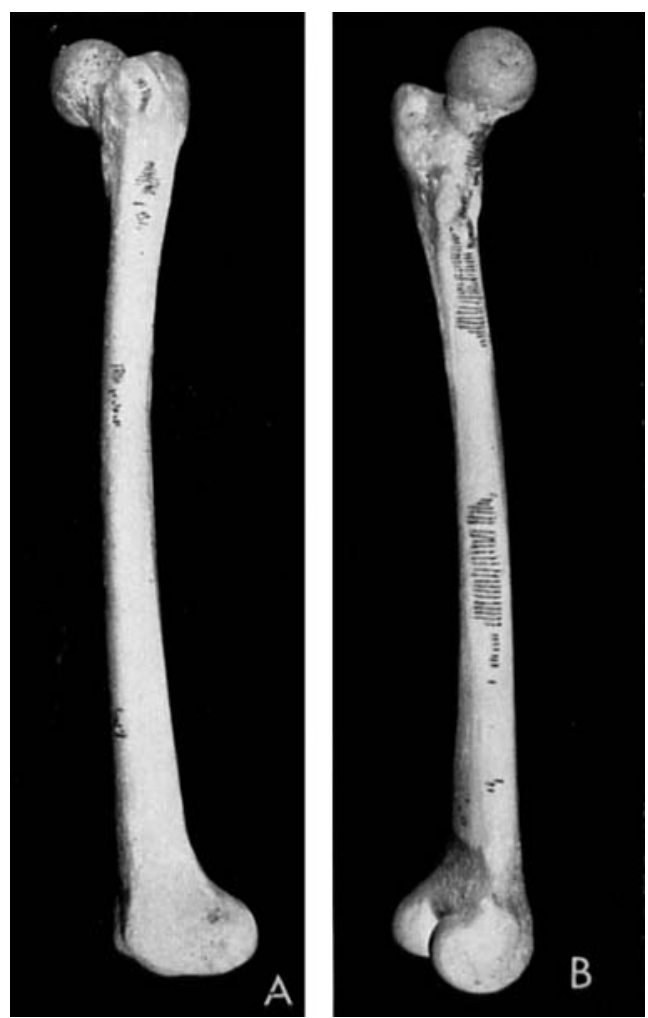

Fig. 2 Areas of tensile (A) and compressive (B) strain produced in a stresscoated human femur by a static (slowly applied) maximum load of $240 \mathrm{lbs}$. (from Evans and Goff, Am. J. Phys. Anthrop., 15, '57).

tude of the maximum load required to produce the stresscoat patterns we illustrated varied from the 125 pounds for the femur of a baboon to 800 pounds for the femur of a gorilla.

Tappen does not distinguish between strain and stress, two fundamentally different concepts in Newtonian mechanics. Strain is the change in the linear dimensions of a body as the result of the application of a force. There are no standard units of measurement for strain (which can be measured in centimeters per centimeter, inches per inch or percentage) and if the strain is sufficiently large it can be seen, e.g., the stretching of a rubber band.

The tendency of a body to be deformed by the application of a force is resisted by an intermolecular force that tends to restore the body to its original dimensions. The ratio of this restoring force to the area upon which it is assumed to act is stress (Dees, '45). Engineers usually define stress as force per unit area (Harris, '63). Because stress is a ratio it can never be seen, no matter how large it is. Stress is always a derived quantity and can only be computed in terms of force (load) per unit area (lbs $/ \mathrm{in}^{2}$ or $\left.\mathrm{kg} / \mathrm{cm}^{2}\right)$. Because stress is a ratio, and hence invisible, splitlines cannot be considered as "lines of stress" or "stress trajectories."

Tappen comments on trajectorial diagrams and states that we "never explain why the elaborately curved parts of the 'trajectories' are not represented by any stresscoat patterns. .." $\mathrm{He}$ is referring to

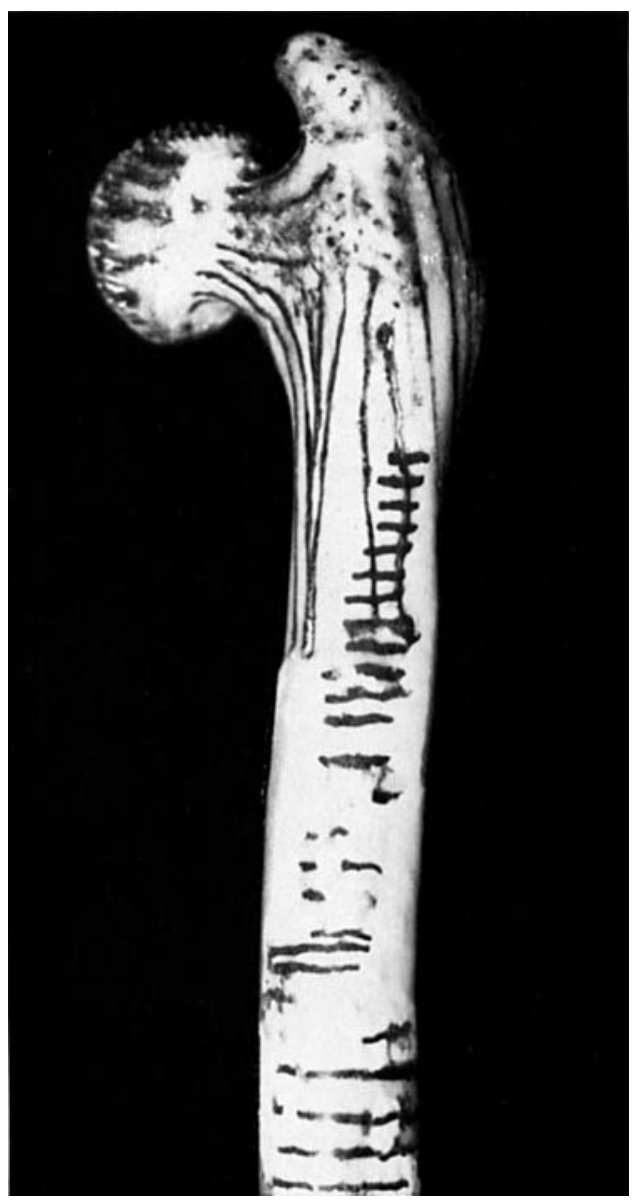

Fig. 3 Split-lines and remnant of stresscoat pattern produced on a baboon femur by a maximum load of $125 \mathrm{lbs}$. (from Evans and Goff, Am. J. Phys. Anthrop., 15, '57). 
our illustration (fig. 4) showing lines we arbitrarily drew on a stresscoated femur simply to illustrate the general nature of trajectories. The reason "the elaborately curved parts" are missing in the stresscoat patterns is that stresscoat patterns are produced by strain, not stress. Consequently, stresscoat patterns do not represent stress trajectories or lines of stress.

The concept of stress trajectories is a technical one in the science of "strength of materials" but frequently the term is used loosely. We clearly stated that "A stress trajectory is defined in mechanics as the curve along which the principal stresses at any point will fall." A trajectorial diagram consists of a number, theo-
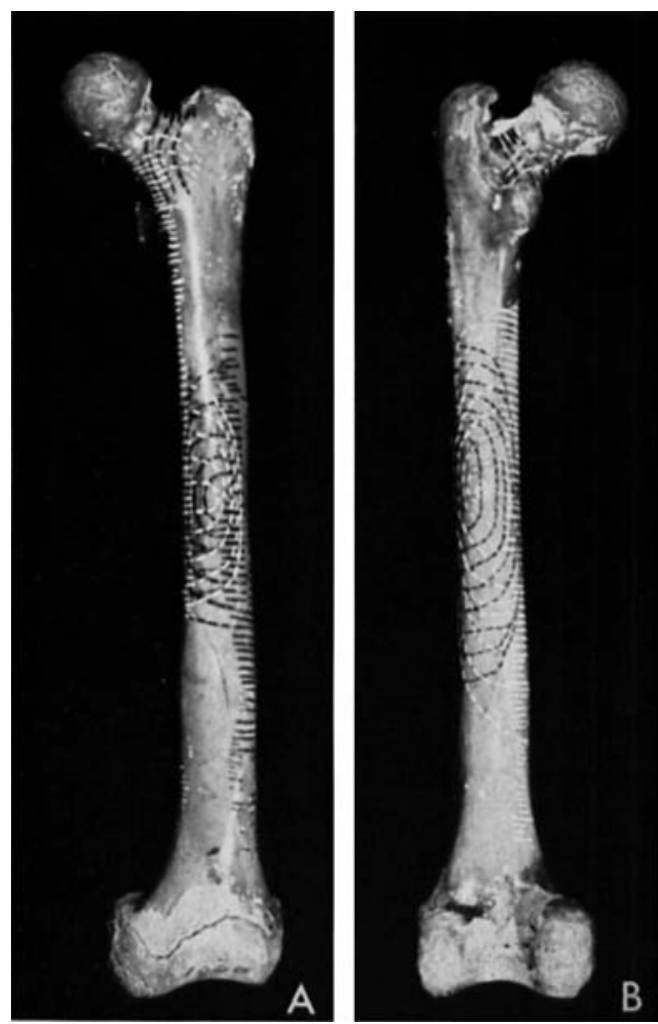

Fig. 4 Areas of tensile strain (solid black lines) and compressive strain (solid white lines) produced in a stresscoated chimpanzee femur by a maximum static load of $200 \mathrm{lbs}$. The tensile trajectories (black broken lines) and the compressive trajectories (white broken lines) were arbitrarily drawn on the bone after the test. See text for discussion (from Evans and Goff, Am. J. Phys. Anthrop, 15, '57). retically infinite, of such curves along which tensile and compressive stresses have been computed for different points. The curves for tensile and compressive stresses, respectively, cross each other at right angles. Most of the stresses in a trajectorial diagram are internal, rather than on the surface as are stresscoat cracks, and the magnitude of the stress at each point on a curve is usually indicated. Furthermore, a trajectorial diagram is flat and two dimensional in nature. Thus, a trajectorial diagram is quite different from a stresscoat pattern or, in fact, any lines drawn on the curved surface of an intact three dimensional bone.

The illustration Tappen refers to (fig. 4) is not a trajectorial diagram of a femur. As we stated, "If the magnitude of the stress were actually computed at various points and these trajectories shown in a trajectorial diagram they would have to cross one another at a $90^{\circ}$ angle. However, a bone is not a plane surface like a trajectorial diagram and in extending the lines from the stresscoat cracks it was difficult to draw them on the curved surface of the bone so that they would cross at a right angle." However, the magnitude of the stress at various points was not computed, the lines drawn on the bone did not cross each other at a $90^{\circ}$ angle, and an intact bone is not a plane surface. Thus, we did not, as Tappen claims, "ignore their own dictum: Stress trajectories or lines of stress' cannot be seen in a bone and a bone is not the type of body for which trajectorial diagrams can be properly drawn."

An attempt to draw a trajectorial diagram of the proximal part of a human femur under a concentrated load was made by Koch ('17). However, Zarek ('59), an engineer, has noted that "... an examination of his results suggests that they are not in agreement with either the theory of stress analysis or the assumptions he made for his analysis."

The belief that a trajectorial diagram could be drawn for a bone is involved in the Trajectorial Theory of bone architecture, according to which the trabeculae in spongy bone lie along stress trajectories. The theory is based primarily upon a trajectorial diagram that Culmann drew of $a$ Fairbairn crane, not an actual bone. In 
discussing the problem Zarek (loc. cit.) emphasizes that he (i.e., Culmann) "based his calculations upon the assumption that his crane was made of homogeneous, isotropic solid material resembling the femur in shape and loading." These conditions are quite different from those in an intact bone, which is composed of heterogeneous, anisotropic, porous material. Furthermore, a femur is a hollow tubular structure. Zarek points out that Culmann's analysis is based "... on two-dimensional stress, although three-dimensional analysis is required" and that Culmann assumed static loading of the femur in the frontal plane whereas "the actual force on the head of the femur is mostly of a dynamic character with its direction of action oscillating appreciably, so that the maximum value of the force does not necessarily coincide with the frontal plane." Zarek also notes that the frontal cross-sectional plane of the femur which excited Culmann's interest "need not be one of the principal planes and that the direction of the two principal stresses need not be parallel to the frontal plane." Zarek thinks "it is difficult to accept the opinions of those who believe in the mechanical adaptation of bone, that is, that the trabeculae lie along the paths of maximum stress within the bone and thus transmit the maximum load with a minimum of material. Other explanations must be sought."

If split-lines do not represent "stress trajectories" or "Iines of stress" what do they represent? The best interpretation of split-lines is that they show the "grain" of a bone as suggested by Dempster and Coleman ('61). A similar interpretation was made by Seipel ('48), a reference cited by Tappen. Seipel wrote that "The coloring lines are by no means trajectories (meaning structural lines of stress), only indicators of the main flow of lamellae and fibrous organization in the bone." (Italics Seipel). In an earlier publication Tappen ('54) himself, when describing split-lines, stated that "Usually splits resulted rather than round holes, because bone has a minute 'grain' analogous to that of wood." Seipel also states: "It seems obvious that in a study of the architecture of a tissue, and especially in the mechanical and functional interpretation of this architecture, extreme caution has to be exerted in the application of mechanical laws." (Italics Seipel).

Split-line patterns are often referred to as "architecture" but the grain of a bone is simply a structural characteristic of the bone; it is no more architecture than is the grain of a piece of wood. The orientation of split-lines is, however, related to the shape of the surface of the bone where they are found. Thus, Dempster ('65), in studying split-line patterns on the human skull, found that cylindrical surfaces have a lengthwise grain transverse to the curvature; trenches have a crosswise grain; basin-like fossae often have a circular or spiral grain; margins of foramina and thin laminae with unsupported borders typically have grain parallel to the edge; and spines and sharp processes have an elongated grain. He also noted that "The cortical bone of the brain case (except for its floor) shows a primarily random grain pattern." As Dempster pointed out, "bone is weaker in tension than in compression and several times weaker across the grain than along the grain. In terms of these physical properties, each of the above grain patterns represents a mechanically strong arrangement of bone."

The question still remains - what is responsible for the grain of bone as indicated by split-line patterns? Our research was limited to stresscoat studies (made by Evans) and split-line studies (made by Goff). However, on the basis of information obtained from the literature we suggested that the orientation of split-lines in bone might be related to vascular canals in bone or to the growth of bone. Tappen objects to both of these suggestions.

Dempster and Enlow ('59) have described 11 tracts of vascular canals in cortical bone of the mandible. The pattern of these canals as shown by India ink filled channels in cleared bone, is similar to (but more complete than) the split-line patterns of Seipel (loc. cit.) and others. They also demonstrated numerous Volkmann's canals extending from the periosteum to deeper canals. As evidence against the suggestion that vascular canals are related to the orientation of split-lines Tappen shows split-line patterns (his fig. 
6) consisting of a series of continuous lines running the length of the middle part of the shaft of the femur of a monkey (Cercopithecus). According to him the split-line patterns "in the periosteal surface of one such femoral fragment" shows that "the presence of Haversian systems is not essential to the production of split-lines in bone." However, all blood vessels in bone do not lie in Haversian canals. The split-line patterns Tappen shows for the middle part of a monkey femur may well be related to the primary, non-Haversian canals which, according to Enlow ('63), "are one of the most common of all types of canals in most vertebrate groups." The fact that these canals are not Haversian canals does not necessarily mean they are not vascular canals. Volkmann's canals, which are vascular canals, are also not surrounded by concentric lamellae of bone.

Tappen's comment "that the presence of Haversian systems is not essential to the production of split-lines in bone" seems to imply that we stated or believe that Haversian systems are essential for split-line patterns. Such is not the case. In fact, we pointed out that Ilberg ('35) produced split-lines in human nasal cartilages and gave an illustration of the patterns he found. The situation in cartilage is quite different from that in bone because there are no Haversian systems and most cartilage is avascular. As far as I am aware the functional significance, if any, of split-lines patterns in nasal cartilages is unknown.

In discussing the growth of bone Tappen writes "The photographs of opposite surfaces of the human ilium and scapulae and the dog scapula show radically different split-line patterns in extensive areas. It is impossible to reconcile these opposed patterns with the hypothesis that they are, in the words of Evans and Goff, 'directional growth indicators.' This would require large areas of the two surfaces of the ilium to grow in directions as different from each other as $90^{\circ}$."

It is obvious that growth of the scapula is not the same on opposite sides because, for example, a spine forms on only one side of the bone! Growth has also been different on opposite sides of the ilium, as is evidenced by the presence of gluteal lines and part of the rim of the acetabulum on the lateral side of the bone while the medial side has the sacral articular facet.

Bone growth and remodeling is a very complicated process, while growth is taking place on one side of a bone resorption may be occurring on the opposite side. As Enlow (loc. cit.) has shown for the mandible of the monkey, growth can occur simultaneously in several different directions on opposite sides of the bone. Furthermore, there can be several different types of bone at the same level of the femoral metaphysis.

I can only assume that Tappen's interpretation that growth should be symmetrical on opposite sides of a bone ignores the century old doctrine that present day forms carry the stamp of their evolutionary origin; limbs from fish to man have been bilateral and there have been lateral and medial surfaces with functional relations to the trunk, to the muscles and to all other parts. There is no more reason why growth must be the same on opposite sides of a bone than there is that differentiation of muscles must be the same on opposite sides of the hand or the foot or that right and left sides of the body be the same rather than mirror images.

The mechanical behavior of a bone or other body when subjected to a force or load is dependent upon the kind, the distribution, the amount and the physical properties of the material composing the body. Stresscoat studies on intact bones loaded in a specific way are concerned with all of the characteristics mentioned. Furthermore, stresscoat has been used on living bones in living animals and it is known that the mechanical behavior of an intact bone, as indicated by stresscoat patterns, outside of the body is similar to that of the same bone in the living body when the experimental conditions are similar (Gurdjian and Lissner, '45). Splitlines can only be obtained on partially decalcified bones. Consequently, the physical properties, strength characteristics and mechanical behavior of the bones would be different from those of the same undecalcified bones in a living body. 
It has been known since the work of Rauber (1876) that bone is an anisotropic material, i.e. a material whose strength is not the same in all directions. For example, Dempster and Liddicoat ('52) found that the compressive strength of both wet and dry cubes of human cortical bone was much greater in the longitudinal direction (parallel to the fibers or grain) than in the radial (surface to marrow) or than in the tangential directions. Evans ('64) reported similar highly significant directional differences in the tensile strength of standardized prisms of wet embalmed human femoral and tibial cortical bone.

Before any mechanical significance can be assigned to split-line patterns it is necessary to know the kind of bone, the distribution of osseous material (i.e., cortex, spongiosa, density, etc.), the amount of bone and the physical properties of the bone where the split-lines are found. All this is irrelevant as long as split-lines are interpreted anatomically as grain. Grain, however, implies different directional properties or anisotrophy.

\section{LITERATURE CITED}

Dees, B. C. 1945 Fundamentals of Physics. The Blakiston Company, Philadelphia, Pa.

deForest, A. V., and G. Ellis 1940 Brittle lacquer as an aid to stress analysis. J. Aeronaut. Sci., 7: 205-208.

Dempster, W. T. 1965 The grain of cortical bone in relation to structural features of the adult skull, Anat. Rec., 151: 342-343.

Dempster, W. T., and R. F. Coleman 1961 Tensile strength of bone along and across the grain. J. Appl. Physiol., 16: 355-360.

Dempster, W. T., and D. H. Enlow 1959 Patterns of vascular channels in the cortex of the human mandible. Anat. Rec., 135: 189-205.
Dempster, W. T., and R. T. Liddicoat 1952 Compact bone as a non-isotropic material. Am. J. Anat., 91: 331-362.

Enlow, D. H. 1963 Principles of Bone Remodeling. Charles C Thomas, Springfield, Ill.

Evans, F. G. 1957 Stress and Strain in Bones. Charles C Thomas, Springfield, III.

Evans, F. G. 1964 Significant Differences in the Tensile Strength of Adult Human Compact Bone. Proceedings of the First European Bone and Tooth Symposium, Oxford, 1963, 319331. Pergamon Press, Oxford, England.

Evans, F. G., and G. W. Goff 1957 A comparative study of the primate femur by means of stresscoat and the split-line techniques. Am. J. Phys. Anthrop., 15: 59-90.

Evans, F. G., and H. R. Lissner 1948 "Stresscoat" deformation studies of the femur under static vertical loading. Anat. Rec., 100: 159-190.

Gurdjian, E. S., and H. R. Lissner 1945 Deformation of the skull in head injury. A study with the "stresscoat" technique. Surg. Gynec. and Obst., 81: 679-687.

Harris, C. O. 1963 Strength of Materials. Revised Edition. Amer. Tech. Soc., Chicago, Ill.

Ilberg, A. 1935 Ueber die funktionelle Architektur der Naselknorpel und ihre knocheren Umgelung beim Menschen. Ztschrf. f. Laryng. Rhim. Otol., 26: 239-257.

Koch, J. C. 1917 The laws of bone architecture. Am. J. Anat., 21: 177-298.

Rauber, A. A. 1876 Elasticität und Festigkeit der Knochen. Engelmann, Leipzig.

Seipel, C. M. 1948 Trajectories of the Jaws. P. A. Norstedt and Sons, Stockholm.

Tappen, N. C. 1954 A comparative functional analysis of primate skulls by the split-line technique. Human Biol., 26: 220-238.

- 1964 An examination of alternative explanations of split-line orientation in compact bone. Am. J. Phys. Anthrop., 22: 1-19.

Zarek, J. M. 1959 The importance of biomechanics as a service to man, illustrated by a discussion of problems in metallic osteosynthesis. Symposium on Biomechanics, London 3-8. The Institute of Mechanical Engineers. 